\title{
Design of a Recommender System (RS) for Job Searching Using Hybrid System
}

\author{
Muhammad Bin Abubakr Joolfoo*, Radhika Dhurmoo, Rameshwar Ashwin Jugurnauth \\ Department of Electrical and Electronic Engineering, University of Mauritius, Reduit, Mauritius

\section{Email address:} \\ muhammad.joolfoo@umail.uom.ac.mu (M. B. A. Joolfoo), radhika.dhurmoo@umail.uom.ac.mu(R. Dhurmoo), \\ r.jugurnauth@umail.uom.ac.mu (R. A. Jugurnauth) \\ ${ }^{*}$ Corresponding author
}

\section{To cite this article:}

Muhammad Bin Abubakr Joolfoo, Radhika Dhurmoo, Rameshwar Ashwin Jugurnauth. Design of a Recommender System (RS) for Job Searching Using Hybrid System. Internet of Things and Cloud Computing. Vol. 8, No. 3, 2020, pp. 31-40. doi: 10.11648/j.iotcc.20200803.11

Received: July 24, 2020; Accepted: December 18, 2020; Published: December 22, 2020

\begin{abstract}
By and large, searching for work while examining a rundown of enlisting positions on enrollment locales, which truly cost a lot of time and cash is an irritating thing to do Although most of the time those jobs are not always suitable with users, or users are not satisfy. By doing this, recruiters waste their time by making sure that they are qualify or not. This paper seeks to address a very important issue on the recruitment process which is about matching jobs seekers with jobs offers. These days, the coordinating procedure between the candidate and the activity offers is one of the serious issue's organizations need to deal with. Short listing candidates and screening resumes are long time-consuming tasks for the company, especially when 80 percent to 90 percent of the resumes received for a role are unquailed. We have designed and proposed a hybrid personalized recommender system used for job seeking and online recruiting websites adapted to the cold start problem using a collaborating predictive algorithm. The hybrid system is composed of Content-Based filtering as well as Knowledge-based Approach which will be has been coded using the Python language. Precise Recommender Systems are very important nowadays.
\end{abstract}

Keywords: Content-Based Filtering, Knowledge-Based Approach, Hybrid-Based Approach Component

\section{Introduction}

The commonness of computerized innovation and quick advancement of "World Wide Web has reformed our general public toward a culture dependent on the estimation of data" [1]. The online conditions, for example, interpersonal organizations and weblogs, have accordingly become a copious data source proving a critical impact on clients' way of life [2, 3]. Similar scenario has occurred in ASNs. A tremendous measure of e-content is created on the web each and every day covering different kinds of scholastic things, for example, news, employments, grants, and meetings. As prescribing the most important data to clients dependent on his/her needs is getting troublesome [4], recommender frameworks (RSs) have risen to lighten above difficulties by furnishing clients with the most relevant items [5].

RSs use the clients' past assessments and collaborations with framework to anticipate potential further likes and interests of their clients [6]. Two most well-known methodologies among RSs are communitarian and substance-based recommender calculations [5-8]. in synergistic calculation, the proposals are made dependent on the things that individuals with comparative inclinations and interests favored beforehand [9], while, in content-based strategies, prescribed things are those with content like recently favored things by an objective client [6]. Since, in community oriented separating procedures, the investigation of the real substance isn't required, they are generally utilized in making expectations to channel any kind of things, for example, text, photos, music and videos [4].

Content-based RSs, then again, examine thing portrayals to discover things that are exceptionally compelling to the client [10]. They embrace an all-around organized system for contrasting client interests and the things' specifications to at last propose the most appropriate thing to an objective client [11]. Albeit content-based proposal techniques settle the new 
things' issues, they despite everything experience the effects of the chilly beginning issue in circumstances when new clients are included [5].

The cool beginning issue is separated into two classifications of cold-start things and cold-start clients [9]. Cold-start things challenge is brought about by new things that should be prescribed to clients while there is insufficient past submitted evaluations about them [2, 3, 4]. Cold-start issue happens when another client who has joined an online situation has introduced only barely any suppositions. In such situations, there is no interaction between the new user and the other ones, and hence this not possible to measure the similarity between them. Subsequently, the recommender frameworks can't make dependable proposals [9]. Both communitarian and substance-based recommender frameworks have an inadequacy identified with cold-start issue [5]. For deal proposals, community-oriented calculations require exacting records of past thing appraisals. Be that as may, in certain spaces where new things exist with no past rating records, communitarian strategies can't work appropriately. Subsequently in synergistic separating approaches, cold-start new things issue happens in such conditions when new things should be prescribed [9].

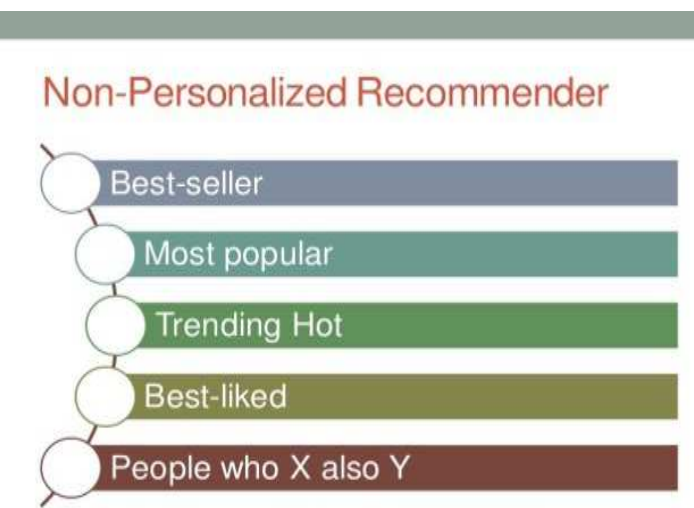

Figure 1. A non personalized system.

This issue has been relieved somewhat by content-based recommender frameworks, which can foresee thing pertinence even without earlier evaluations. Subsequently, the recommender frameworks can't make dependable proposals. "They are unable to recommend items to new users in the absence of any history of previous interactions with the system" [5].

To mitigate the above-mentioned problems, an enhanced version of the content-based algorithm is proposed in the current research whereby social networking techniques are utilized to not only solve the cold-start issue yet in addition improve the expectation precision of the suggestion procedure. This model considers the interests and inclinations of clients' companions and workforce mates notwithstanding clients' own inclinations. In this novel approach, the interests and preferences of users are stored in a hierarchy tree structure. The subsequent sections are organized as follows. The following area presents some related research works in taking care of the cool beginning issue.

\section{Literature Review}

Recommender frameworks are the product devices and strategies that give recommendations. "The main goal of recommender systems is to provide suggestions to online users with a highly relevancies to items so that they can make better decisions from many alternatives available over the Web" [7].

\subsection{Recommendation Problem}

Let $U=\left\{u 1, u 2 \ldots, u_{m}\right\}$ a set of all users and let $=\{1,2, \ldots, n\}$ a set of all possible items that can be recommended such a job offers, jobs seekers. Here the space can be equal to the set $U$ and it can be very large.

The recommendation problem can be formulated [2] as follows:

Let $\mathrm{f}$ be a utility function that measures the usefulness of item to user $\mathrm{u}$,

$$
\mathrm{f}: \mathrm{U} \times \mathrm{I}-\rightarrow \mathrm{R}
$$

where $\mathrm{R}$ is a totally ordered set (e.g the set of ranking recommendation). Then for every user $u \in U$, we want to predict an item $i J \in I$ that maximizes the user utility function. In other words:

$$
\forall u \in U, i^{J}=\operatorname{argmax} f(u, i)
$$

\section{$i \in I$}

Depending to the predictive algorithm used, the input to a RS belongs to one of the subcategories [2]:

Ratings (also called votes), which give the opinion of users on items Demographic data, which refer to user's information's, sometime difficult to get. It is normally collected explicitly from the user iContent data, based on contention analysis The yield of a recommender framework can be [2] a expectation communicated as a numerical esteem or can be a proposal in the event that it is communicated in thing of a rundown of $\mathrm{N}$ positioned things, where $\mathrm{N} \leq \mathrm{n}$. The following part reviews the state of the art of the main approaches to designing RSs that address the problems caused by information overload. Background of recommender systems There exist various calculations and strategies to build recommender frameworks ready to do customized proposals or non-customized suggestions.

\subsection{Non-personalized Recommended System}

In [10], the most straightforward beneficiary easiest structure, customized proposals are offered as positioned arrangements of things. In performing this positioning, RSs attempt to anticipate what the most reasonable items or administrations, depend on the client's inclinations and limitations. So as to finish such a computational task, RSs gather from clients their preferences, which are either unequivocally communicated, e.g., as appraisals for items, or are induced by deciphering client activities. For example, a RS may consider the route to a specific item page as a verifiable indication of inclination for the things appeared on that page. 
RSs improvement started from a fairly straightforward perception: people regularly depend on suggestions gave by others in making standard, day by day choices $[60,70]$. For instance it isn't unexpected to depend on what one's friends prescribe while choosing a book to peruse; managers rely on suggestion letters in their enlisting choices; and while choosing a film to watch, people will in general peruse and depend on the film audits that a film pundit has composed and which show up in the paper they read. In trying to impersonate this conduct, the first RSs applied calculations to use proposals created by a network of clients to convey suggestions to a functioning client, i.e., a client searching for recommendations. The suggestions were for things that comparable clients (those with comparative tastes) had loved. This methodology is named community oriented filtering and its reason is that on the off chance that the dynamic client concurred in the past with certain clients, at that point different proposals originating from these comparative clients ought to be significant too and important to the dynamic client. As internet business Web locales started to create, a squeezing need rose for giving suggestions got from filtering the entire scope of accessible other options. Clients were finding it very difficult to show up at the most fitting decisions from the immense severity of things (products and services) that these Web locales were advertising. The unstable development and assortment of data accessible on the Web and the quick presentation of new e-business administrations (purchasing items, item examination, sell off, and so forth.) much of the time overpowered clients, driving them to settle on poor decisions. The accessibility of decisions, rather than delivering a benefit, began to diminish clients' prosperity. It was gotten that while decision is acceptable, progressively decision isn't in every case better. To be sure, decision, with its ramifications of opportunity, self-rule, and self-determination can get inordinate, making a feeling that opportunity may come to be viewed as a sort of hopelessness instigating oppression.

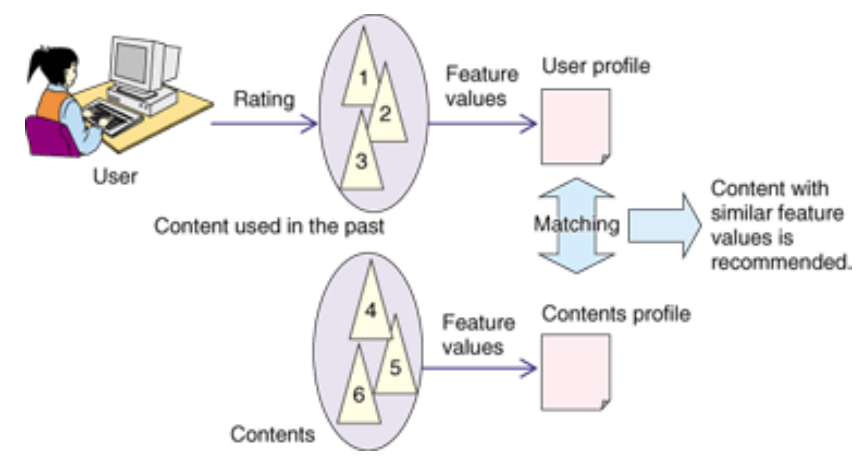

Figure 2. Schema for content-based filtering.

The accuracy of the recommendation increases with the quality of information's provided by users. According to [8], content-based separating is utilizing the strategy to examine a lot of reports and depictions of things recently evaluated by a client, and afterward fabricate a profile or model of the clients intrigues dependent on the highlights of those appraised things. model of the clients intrigues dependent on the highlights of those appraised things. This modeling user approach is called content-based learning and take into account the fact that user's behaves or do not change through time, then the content of past client activities might be utilized to anticipate the deal substance of their future activities. As an example, we can suppose that, if a user has positively rated a song that belong to jazz, then the system can learn to recommend other songs from the same music genre. in order to come to terms with the ever-increasing amount of information while Information recovery is discovering material (normally reports) of an unstructured nature (usually message) that fulfills a data need from inside enormous collections (usually put away on computers). Although, $\mathrm{R}$ has noteworthy contrasts with recommender systems, however, content-based algorithms are driven from R [12]. Some R techniques such as Boolean retrieval, Rocha's algorithm or correlation-based schemes, Probabilistic carter evil systems, Natural language query are useful for recommender systems and can be used in content-based filtering. Other techniques for content-based recommendation use Pattern Recognition/Machine Learning approaches such as Bays and classifiers [13], clustering methods, decision trees, and artificial neural networks. The questions we have to take into consideration while building content-based recommendation system are as follow [7]:

a) How would we make closeness between things?

b) How would we make and update client profiles persistently?

Content based techniques (CBF) are used in some recommender systems applications to provide recommendations to users.

This system doesn't think about the client's local inclinations. Consequently, doesn't require a huge client gathering's inclination for things for better suggestion precision. it just thinks about the client's past inclinations and the properties/highlights of the things. So, compared to collaborative filtering, there are some advantages and drawbacks of content-based filtering that we could note $[14,15]$.

Regarding the advantages, client autonomy Uses the thing's substance to anticipate the client's advantage, no requirements other clients' appraising to discover the comparability between the clients and give the recommendations.

Precise technique: suggestion quality improve as the audit/things content information cumulates.

Ability to suggest clients with one of a kind taste.

1. Capacity to suggest new and unpopular items

2. Straightforwardness: content-based technique can reveal to you they suggest you the things dependent on what highlights, all together word it can give you clarification to proposals.

\section{Methods \& Materials}

The least complex and unique usage of this methodology prescribes to the dynamic client the things that different clients with comparable tastes preferred before. The comparability in taste of two clients is determined dependent on. This is the motivation behind why alludes to communitarian filtering 
"individuals to-individuals correlation." Collaborative filtering is viewed as the most well-known and generally actualized strategy in RS. Part 4 presents a far-reaching overview of neighborhood-based strategies for shared filtering. Neighborhood techniques center around connections between things or, on the other hand, between clients. A thing approach models the inclination of a client to a thing dependent on evaluations of comparative things by the same user. Closest neighbors' techniques appreciate impressive prominence because of their straightforwardness, efficiency, and their capacity to deliver precise and customized suggestions. The creators will address the crucial decisions that are required while executing an area-based recommender framework and give commonsense data on the most proficient method to settle on such choices. At last, the section manages issues of information sparsity and constrained inclusion, regularly saw in huge business recommender systems. A scarcely any arrangements to overcome these issues are introduced. Part 5 presents a few ongoing expansions accessible for building $\mathrm{CF}$ recommenders. Specifically, the creators talk about dormant factor models, for example, network factorization (e.g., Singular Value Decomposition, SVD). These strategies change the two things and clients to the equivalent idle factor space. The inactive space is then used to clarify appraisals by portraying the two items and clients in term of variables naturally deduced from client criticism. The creators explain how SVD can deal with extra highlights of the information, including verifiable input and transient data. They additionally portray strategies to address weaknesses of neighborhood methods by proposing progressively thorough definitions utilizing worldwide advancement techniques. Utilizing such procedures makes it conceivable to lift the breaking point on neighborhood size and to address verifiable input and fleeting dynamics. There suiting accuracy is near that of network factorization models, while offering various viable preferences.

\subsection{Model-based CF Methods}

Is the creators propose to assemble a model of the earth dependent on three measurements: framework clients; the qualities of the information; and the general application. The recommender framework configuration will be founded on this model. The creators represent their rules and the model on a news proposal framework that they have created. At the end of the day, we separate some data from the dataset, and utilize that as a "model" to make proposals without utilizing the total dataset unfailingly. This methodology conceivably offers the advantages of both speed and adaptability.

\subsection{Knowledge-based Approach}

This kind of frameworks endeavors to propose objects dependent on surmising about client's needs and inclinations. This methodology helps clients in the assurance of appropriate arrangements from complex item and administration collections. These arrangements dependent on abusing profound information about the item area learning progressively precise profiles that contain references to ideas defined in outer information bases. The principle inspiration for this methodology is the test of giving a recommender framework the social and phonetic foundation information which are acetizes the capacity of deciphering regular language archives and thinking on their substance. In this section, are perspective on the principle procedures embraced to present some semantics in the proposal procedure is introduced. The portrayal of these procedures is completed by considering a few rules:

1. The kind of information source included (for example vocabulary, philosophy, and so on.);

2. The strategies embraced for the explanation or portrayal of the things;

3. The kind of substance remembered for the client profile;

4. The thing profile coordinating system.

\subsection{Hybrid Approach}

Both substance-based sifting and collective separating have these qualities and shortcomings. Three explicit issues can be recognized for content-based separating:

1. Content portrayal. In specific regions delivering a significant depiction of the substance can be incredibly problematic. In zones where the things contain music or video for example a depiction of the substance isn't continually possible with the current development.

2. Over-specialization. A substance-based filtering structure won't select things if the past customer lead doesn't offer verification to this. Additional techniques must be added to enable the system to make proposition outside the degree of what the customer has quite recently demonstrated eagerness for.

3. Abstract space issue. Content-based isolating systems experience issues in perceiving theoretical information, for instance, reasons for viewpoints and intelligence.

A community oriented sifting framework doesn't have these weaknesses. Since there is no necessity for a depiction of the things being proposed, the system can deal with any kind of information. Additionally, the system can recommend things to the customer which may have a through and through various substance from what the customer has demonstrated to be keen on previously. At last, since proposals depend on the assessments of others it is appropriate for abstract areas like workmanship. Be that as it may, shared separating introduces certain issues of its own:

1. Early issue. Helpful isolating structures can't offer proposition to new things since there are no customer assessments on which to base a gauge. Whether or not customers start rating the thing it will require some venture before the thing has gotten enough examinations in order to make exact proposals. Basically, recommendations will in like manner be mistaken for new customers who have assessed scarcely any things.

2. Sparsity issue. In various information territories the present number of things outperforms the total an individual is proficient (and prepared) to explore by far. This makes it slippery things that are assessed by enough 
people on which to base figures.

3. Dim sheep. Social events of customers are required with covering characteristics. Whether or not such social affairs exist, individuals who don't dependably agree or vary with any get-together of people will get mistaken proposals.

A framework that consolidates content-based separating and community-oriented sifting could exploit from both the portrayal of the substance just as the similitudes among clients. In spite of the fact that there are a few manners by which to consolidate the two methods a qualification can be made between two premise draws near. A half and half methodology consolidate the two sorts of data while it is additionally conceivable to utilize the suggestions of the two separating procedures autonomously.

\section{Job Recommended Framework}

Late investigates show that the expanding requests of IS innovations for human asset the board when all is said in done and enrolling forms specifically. Most organizations put the emphasis on their own e-enlisting stages as essential enrollment channels. Occupation advertisements are distributed naturally hands on entry when they are gone into the framework. Then again, the candidate makes a profile to apply it for one of the recorded activity positions. The client profile is put away in the framework, letting the candidate reuse it for other employment position. The last usefulness gives the organizations plausibility to make the candidates pool. Along these lines, the organizations accomplished a uniform view for all applicant is ${ }^{\text {ee }}$ information in one up-and-comer pool. This pool is utilized by the enlistment.

Division to discover the candidate archives. Proper applicant is ${ }^{\text {ee }}$ records are encouraged to the human asset working environments though the all the all the more taking couldn't care less of. Likewise, the framework bolsters all necessary correspondence forms just is tracks candidate status inside the application procedure.

The e-enrolling stages are typically founded on Boolean pursuit and sifting strategies "that can't adequately catch the intricacy of an individual activity fit as determination choices. Numerous literary works have been applied the recommender framework deal into the activity issue. Malinowski et al. (2008i) verified that, we should consider unary qualities, for example, singular aptitudes, mental capacities and character that control the fit between the individual and the undertakings to be practiced, as well as the relational attributes that determine the fit between the individual and the upcoming team members. Right now, ordinarily recognize (1) individual employment, (2) man group and (3) man association fits [21]. Hence, the enlistment approach must cover these angles. "Keim argues that transferring recommender system approach to search for persons is a challenging but promising goal" [4].

Along these lines, numerous proposal approaches went after coordinating applicants and positions to beat the past difficulties of all-encompassing e-selecting stages.

\subsection{System Requirements for Candidates/Jobs Recommendation}

There are significant necessities introduced in literary works that ought to be determined when suggesting possibility for a particular activity [21].

1. The matching of individuals to job depends on skills and abilities that individuals should have.

2. Recommending people is a bidirectional process that Needs to take into account the preferences not only of the recruiter but also of the candidate.

3. Recommendations ought to be founded on the applicant traits, just as the social angles that decide the fit between the individual and the colleagues with whom the individual will be worked together.

4. Individual is viewed as one of a kind; we can't pick a solitary individual a few times, for example, a film or book.

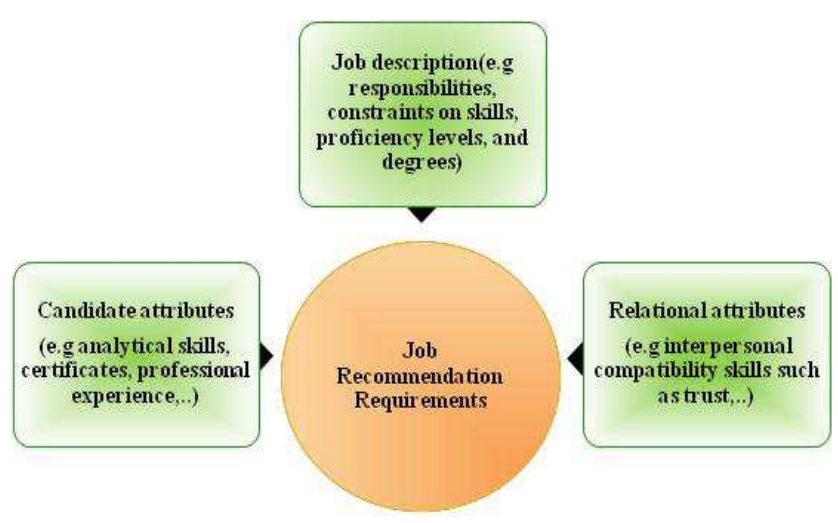

Figure 3. Model of system requirements for candidates/job recommendation.

"Job recommendation problem is bidirectional recommendation between job-seeker and job. The recommendation process can be divided into two parts: job recommendation and job-seeker recommendation. The design idea of these two parts is the same roughly [21]. For an occupation searcher, the activity with higher coordinating degree ought to be prescribed to him. Correspondingly, for a vocation, the activity searcher with higher coordinating degree ought to be prescribed to it

As a rule, the positioning things either are the top in applicants that best fit the activity in thought or the top in work profiles that best fit the candidates' inclinations. Furthermore, Fazel-Zarandi and Fox referenced that aptitudes prerequisites coordinating need to recognize must-have and wonderful to-have necessities in the organizing technique. Must-have necessities are limitations that ought to be controlled by the candidate, though pleasant to-have prerequisites are inclinations that are thought about when positioning candidates. Figure 3 sums up the activity proposal prerequisites in a bound together model.

\subsection{Job Recommendation Information}

Jobs and candidates should be matched based on some important factors used as indicators of performance on the job. 
In choice hypothesis, the accessible data at a specific time of the choice determination is called indicator information which contains the individual qualities. The real determination strategy is called indicator. The expectation procedure $\mathrm{s}$ alluded to the appraisal of the criteria utilizing the indicator information and a technique explicit method for information mix.

Be that as $\mathrm{t}$ may, to develop applicant profiles, the meta-information separated from resumes. Beam and Smyth proposed a framework that assembles client profile in enlistment condition legitimately from dissecting the practices of web clients. Right now, profiles are developed by latently identifying the snap stream and read-time conduct of clients. Malinowski et al. an information for their CV-framework: demo-realistic information, instructive information, professional training, language abilities and $\mathrm{T}$ aptitudes, grants, distributions, others. In general, candidate's profile is composed of three sections [21].

1. The main section of the suggestion clarifies the reason for the activity, just as your own subtleties with Name, Skills and capabilities and past experience of the work.

2. The suggestion letter contains explicit data on the individual you are expounding on, including why they are qualified, and what they can contribute. On the off chance that important, utilize more than one section to give subtleties. Incorporate explicit models verifying the individual's capabilities at whatever point conceivable.

3. This area of the suggestion letter contains a concise synopsis of for what reason are you are suggesting the individual. Express that you "energetically suggest" the individual or that you "suggest without reservation" or something comparable.

The closing section of the suggestion letter contains a proposal to give more data. You can incorporate a telephone number inside this passage. Another choice is to incorporate a telephone number and email address in the arrival address area or mark of the letter.

\section{Job Recommendation Architecture}

Suggestions are a coordinating issue. Given a lot of clients and a lot of things, we need to coordinate clients to their favored things. There are two significant level ways to deal with this sort of coordinating: content based and conduct based. They each have upsides and downsides, and there are additionally approaches to consolidate them to exploit both techniques. Content-based methodologies use information, for example, client inclinations and highlights of the things being suggested, to decide the best matches. For suggesting employments, utilizing catchphrases of the set of working responsibilities to coordinate watchwords in a client's transferred continue is one substance-based methodology. Utilizing catchphrases in a vocation to secure other comparative looking positions is another approach to actualize content-based recommendations. Behavior-based methodologies influence client conduct to produce proposals. They are space freethinker, which means similar calculations that take a shot at music or films can be applied to the employments area. Conduct based methodologies do experience the ill effects of a virus start issue. In the event that you have little client movement, it is a lot harder to create great quality proposals. Half and half frameworks presented in this paper depend on the processing of similitudes between things, assuming that comparable things have comparable appraisals for a given client. We propose to register likenesses in two unique manners: an innocent similitude between two arrangements of highlights, and a managed closeness so as to recognize closest neighbors concerning important highlights. Important highlights are those which are contributing in clarifying employment board returns. At the point when another thing must be appraised for all clients, his closeness as for every single past thing is processed.

\section{Contextual Investigation: A Case of Prescribing Contender for Explicit Occupation}

So as to comprehend the activity suggestion issue, we present a straightforward and solid model for coordinating up-and-comer with work necessities. We focus on measurable skills possessed by human resources. This model applies a substance-based suggestion approach that utilized the credits identified with both employment and up-and-comers. As mentioned before in content-based, we must construct a profile for each item, which is a record representing the important features of that item. For simplicity, we consider only few features that might be relevant to a recommendation system. The undertaking of an occupation recommender framework is to recover a rundown of candidates' CVs for a new position. We direct this model utilizing one expected set of responsibilities and rundown of 5 forthcoming up-and-comers CVs. The job description was downloaded from Careers portal website2:

1. Employment title: Computer framework director.

2. Expected set of responsibilities: The planned representative will screen, work and regulate the inside PC frameworks of an association.

The candidates ${ }^{\text {ec }} \mathrm{CV}$ s were downloaded from BSR site 3 . As referenced over, the initial step to decide the best fit among up-and-comers and set of working responsibilities is building the activity profile and the planned employee's profiles. We separate a few highlights from representative continues and expected set of responsibilities to assemble the two profiles. At that point, we gauge this model of parameters by making rating lattice $\mathrm{Rx}, \mathrm{y}$, where $\mathrm{x}$ speaks to the activity and y speaks to the up-and-comer CVs.

The rating network $\mathrm{Rx}, \mathrm{y}$ changed by treating the estimations of applicant's qualities as appraisals of the considerable number of properties extricated from the resumes utilizing any comparability measures. That implies the activity profile just "as the candidates profiles spoke to as vectors. We applied three measures right now: Similarity, Euclidean Distance and New Jaccard Measure [22]. The profiles vectors are developed as the 
accompanying: (BSc required), (MSc into required), (if one of these majors: Software Engineering, Computer Programming or IT), (if he/she worked in IT sector), (if the candidate's experience more than 4 years), 1-3 (for English skill levels), 1 (if the candidate has Oracle developer skill).

The resultant job's vector is [ $\left[\begin{array}{lllllll}0 & 1 & 1 & 1 & 1 & 3 & 1\end{array}\right]$ and the resultant candidates' vectors are:

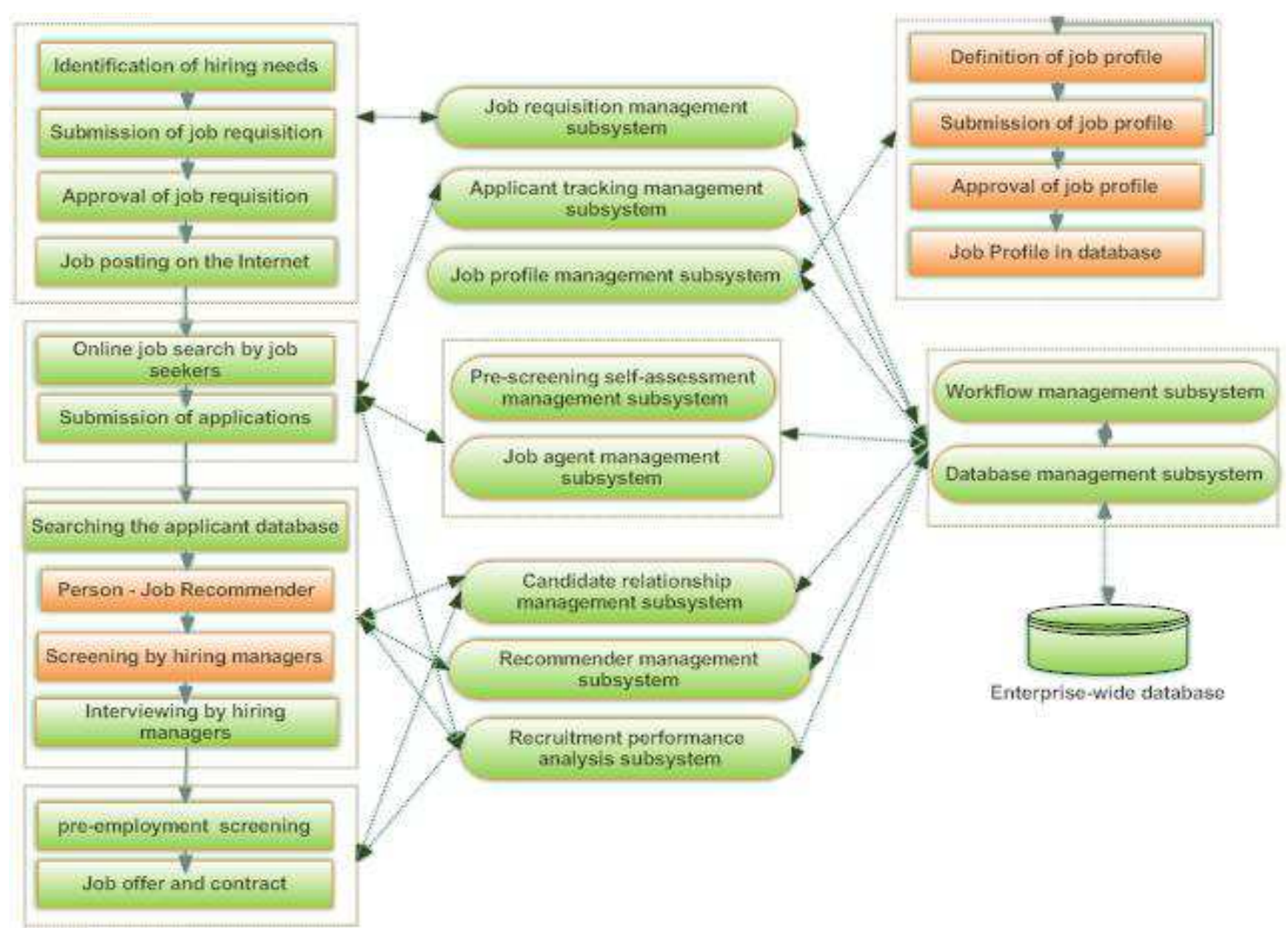

Figure 4. Complete architecture of job recommender system (RS).

Table 1. Ranking of candidates for the job position using three similarity measures.

\begin{tabular}{lll}
\hline Cosine Similarity & Euclidean distance & New measure \\
\hline $3^{\text {rd }}$ person 0.99 & $3^{\text {rd }}$ person 1.0 & $3^{\text {rd }}$ person 0.94 \\
$2^{\text {nd }}$ person 0.82 & $2^{\text {nd }}$ person 1.41 & $2^{\text {nd }}$ person 0.83 \\
$4^{\text {th }}$ person 0.81 & $4^{\text {th }}$ person 1.7 & $4^{\text {th }}$ person 0.78 \\
$1^{\text {st }}$ person 0.70 & $1^{\text {st }}$ person 2.0 & $1^{\text {st }}$ person 0.61 \\
$5^{\text {th }}$ person 0.67 & $5^{\text {th }}$ person 2.65 & $5^{\text {th }}$ person 0.48 \\
\hline
\end{tabular}

1st person: [ [ $\left.\begin{array}{lllllll}0 & 0 & 1 & 0 & 0 & 2 & 1\end{array}\right]$, 2nd person: [ $\left[\begin{array}{lllllll}0 & 1 & 0 & 1 & 1 & 3 & 0\end{array}\right], 3^{\text {rd }}$ person: [ $\left[\begin{array}{lllllll}0 & 1 & 1 & 1 & 1 & 2 & 1\end{array}\right]$, 4th person: [ [ $\left.\begin{array}{lllllll}0 & 1 & 0 & 1 & 1 & 2 & 0\end{array}\right]$, and 5th person: $\left[\begin{array}{lllllll}1 & 1 & 0 & 1 & 1 & 1 & 0\end{array}\right]$. The candidates' ranking after applying the Cosine Similarity, new Jaccard measure is introduced in Table 1 .

This model plans to discover an up-and-comer who best fits the necessities of occupation profile $\left[\begin{array}{llll}0 & 1 & 3 & 1\end{array}\right]$. in light of the three closeness measures, third individual's the best applicant who fits work prerequisites, trailed by second individual and fourth individual.

The 1st person and 5th person are the least appropriate candidates for the job requirements.
Notwithstanding, applying the Boolean quest technique for this issue will choose up-and-comers who have explicit catchphrases in their profiles yet will neglect to consider the degree of exactness in specific assignments. It will overlook the evaluations of aptitudes.

\section{Job Recommendation Technique}

As of late, a few recommender framework systems applied $\mathrm{n}$ applicants/work coordinating issue, began by the staff choice methodology that proposed by "Färber et al. who built up a probabilistic cross variety suggestion approach for competitors/work arranging. At that point, their model used and stretched out by Malinowski et al. and Keim [21].

\subsection{Mixture Recommender Systems / Hybrid Rs}

These RSs depend on the blend of the previously mentioned strategies. A cross breed framework consolidating strategies An and $B$ attempts to utilize the benefits of $A$ to fix the hindrances of $\mathrm{B}$. For example, $\mathrm{CF}$ techniques experience the 
ill effects of new-thing issues, i.e., they can't suggest things that have no evaluations. This doesn't restrict content-based methodologies since the expectation for new things depends on their depiction (includes) that are normally effectively accessible. Given (at least two) fundamental RSs methods, a few different ways have been proposed for consolidating them to make another mixture framework (see for the exact depictions). As we have just referenced, the setting of the client when she is looking for a proposal can be utilized to all the more likely customize the yield of the framework. For instance, in a fleeting setting, excursion suggestions in winters should be very not the same as those gave in summer. Or on the other hand an eatery proposal for a 14 Francesco Ricci, Lior Rokach and Bracha Shapira Saturday evening with your companions ought to be unique in relation to that recommended for a workday lunch with collaborators. Section 7 presents the general thought of setting and how it tends to be displayed in RSs. Examining the potential outcomes of joining a few setting mindful proposal procedures into a solitary unified approach, the creators likewise give a contextual investigation of one such consolidated methodology. Three diverse algorithmic standards for consolidating logical data in to their acclamation processed discussed: decrease based (pre-filtering), relevant post filtering, and setting displaying. In reduction-based (pre-filtering) strategies, just the data that coordinates the present utilization setting, e.g., the appraisals for things assessed in a similar setting, are utilized to figure the suggestions. In relevant post filtering, the suggestion calculation overlooks the setting data. The yield of the calculation is filtered/changed in accordance with incorporate just the suggestions that are important in the objective setting. In the logical displaying, the more modern of the three approaches, setting information is unequivocally utilized in the expectation mode.

\subsection{A System for Screening Candidate}

Singh et al. have promoted the PROSPECT structure, define determination help mechanical assembly helping scouts to shortlist up-and-comer resumes list. It mines resumes to get rid of features of contender profiles, as an example, capacities, preparing, and knowledge. It used information recuperation frameworks to rank possibility for a given action position. for each movement profile, the structure positions contenders reliant on the similarity between work profile and up-and-comers resumes. The situating is often refined by including isolating standards/ filtering criteria. These criteria based on the candidate meta-data, as well as on the information that is automatically extracted from the candidate resumes. This technique includes three guideline sections: Batch processor, Query processor and Resume matcher. New applications are from the outset arranged by the pack processor. It hides away up-and-comer meta-data within the essential database and concentrates data from the contender resumes, which along these lines saved in isolated database. This data is employed by the question processor and therefore the resume matcher to offer the positioning applicant rundown to a given client inquiry.

\section{Reciprocal Recommendation for Recruitment}

So as to take care of the issue that the conventional suggestion calculation can't complete the two routes suggestion among client and things, by presenting the idea of complementary proposal calculation dependent on bi-directional coordinating is proposed. Most importantly, contingent upon the particular essential of the resume conveyance on enrollment framework and mining the potential inclination data in figuring similitude, a comparability estimation strategy is proposed. Finally, the complementary proposal is accomplished by the corresponding an incentive from high to low. The exploratory outcomes show that the proposed calculation cannot just take care of the issue of two route suggestion between work searchers and employments, yet additionally improve the exactness of proposal.

\section{Discussion}

The half and half activity suggestion approaches introduced consolidated at least two strategies to beat the issues that experience the ill effects of utilizing every procedure independently. For instance, while the likelihood half and half methodologies in section A, understood a bidirectional proposal and attempted to cover distinctive determination measurements, they have to improve by including more highlights for people and stretching out by different social perspectives other than trust. Besides, "they conceivably got the equal depiction with Yes and No when state customer tendencies, and it can't check the degree of customers tendencies for each record well, so the idea of proposition isn't high [21].

With respect to the substance-based occupation recommender frameworks, it is introduced a few methodologies and frameworks dependent on CBF procedures. As referenced in the $\mathrm{CBF}$, it is restricted by the highlights that expressly connected with prescribed articles. In this manner, since the applicants' resumes are normally spoken to by the most significant highlights utilizing catchphrases, frameworks can't recognize various watchwords meaning. Also, the issue generally connected with the unadulterated CBF frameworks; it can't prescribe occupations that are not the same as anything the client has seen previously. Employments will be prescribed on the off chance that they are like different occupations that the candidate has just intrigued. Along these lines, the candidates need to rate an adequate number of employments before a $\mathrm{CBF}$ recommender framework can extremely solid proposals. For instance, recommender framework in above forms a mechanized framework to suggest occupations for candidates dependent on their past activity narratives. this technique is employed a classifier that creates a recommendation by means of setting them abreast of content information. It experienced adaptability and knowledge inadequately issues. Notwithstanding, this framework plays out the suggestion an unary connection and 
disregards the individual item fit when matching candidates with jobs. Then again, the applicants can furious past observed employment profiles to be coordinated with content-based separating. Along these lines, a strategy dependent on collective separating would likewise bomb because of a too inadequately filled grid of practically identical appraisals. Also, in abilities prerequisites coordinating, we are keen on deciding if an individual fulfills a lot of necessities. Most huge essentials are hard goals however perfect necessities are fragile prerequisites that are viewed as when situating. Therefore, the determination of possibility to occupations needs to coordinate unary up-and-comer characteristics just as social data and fuse up-and-comer appraisals for as of now observed jobs' profiles to build up a computational model that reasonable for these necessities. We acknowledge that this region of research has critical sensible consequences in different degrees of e-selection process that can support boss and spotters. This isn't expected to supplant totally conventional choice strategy yet intends to help the human asset office by a rundown of applicants from which picked the reasonable up-and-comer.

\section{Conclusion}

In this article, we utilized an article investigation of various diaries and procedures identified with the choosing procedure and therefore the activity proposal looks into. we've seen from our writing survey and from the difficulties that confronted the all-encompassing e-enlisting stages, an expanded requirement for improving the character of competitors/work coordinating. The recommender framework advancements achieved huge accomplishment in an expansive scope of utilizations and possibly a ground-breaking looking and suggesting methods. Thusly, there's an outstanding open entryway for applying these advancements in selection condition to enhance the design quality. This overview shows that few methodologies for work suggestion are proposed, and various strategies consolidated so on deliver the simplest fit among occupations and competitors. We introduced best in school of employment suggestion even as, an identical report for its methodologies that proposed by written works. Moreover, we surveyed commonplace recommender framework systems and therefore the selecting procedure related issues. We reason that the sector of occupation suggestions is so far unripe and need further enhancements. As a big a part of our persistent research, we hope to fabricate another proposition Enhance and test with data for specialist and staffing data from enormous associations. Notwithstanding, we decide to pre-create the likeness quantifies that reasonable for this recommendation.

\section{References}

[1] Jayavardhana Gubb, Rajkumar Buyya, Slaven Marusic, and Marimuthu Palaniswami. internet of things (iot): A vision, architectural elements, and future directions. Future generate on computer systems, 29 (7): 1645-1660, 2013.
[2] Aristomenis Lampropoulos and George A Tsihrintzis. Machine Learning Paradigms: Applications in Recommender Systems, volume 92. Springer, 2015.

[3] Gediminas Adomavic us and Alexander Tuzhilin. Toward the next generation of recommender systems: A survey of the state-of-the-art and possible extensions. EEE transactions on knowledge and data engineering, 17 (6): 734-749, 2005.

[4] James Bennett, Stan Lanning, etial. The netflix prize. in Proceedings of KDD cup and workshop, volume 2007, page 35. NewYork, NY, USA, 2007.

[5] Abhinandan S Das, Mayur Datar, Ashutosh Garg, and ShyamiRajaram. Google news personalizaition: scalable online collaborative filtering. in Proceedings of the $16^{\text {th }}$ nternational conference on World Wide Web, pages 271-280. ACM, 2007.

[6] James Davidson, Benjamin Liebald, Junning Liu, PalashiNandy, Taylor VaniVleet, Ullas Garg, Sujoy Gupta, Yu $\mathrm{He}$, Mike Lambert, Blake Livingston, et al. The youtube video recommendation system. in Proceedings of the fourth ACM conference on Recommender systems, pages 293-296. ACM, 2010 .

[7] Suresh K Gorakala and M chele Usuelli. Building a recommendation system with R. Packt Publishing Ltd, 2015.

[8] Francesco, Lior Rokach, and Bracha Shapira. ntroduction to recommender systems hand book. in Recommender systems handbook, pages 1-35. Springer, 2011.

[9] Jul e S'egu'ela. Fouille de donn'eesitextuelles et system's de recommendation appliqués aux off resid employ diffuses surile web. PhD thesis, Paris, CNAM, 2012.

[10] Anil Poriya, Tanvi Bhagat, Neev Patel, and Rekha Sharma. Non-personalized recommender systems and user-based collaborative recommender systems.

[11] Alexandr Singleton. Recommender systems: Collaborative filtering and content-based recommending. http://slideplayer.com/slide/5692490/, 2016.

[12] D. Jannach, Felfernig A. Zanker, M., and G. Friedrich, editors. Recommender systems: an ntro-duction. Cambridge University Press, 2010.

[13] M. Pazzani and D. Billsus. Learning and revising user profiles: the dentification of nteresting websites. Mach. Learn., 27 (3): 313-331, 1997.

[14] R chard Steinmetz David, Domingo Gregory, Liu A ko, and Tzu-Yu Chen Amy. The restaurant dilemma: Personalized recommendations for groups of people. https://nycdatascience.com/blog/istudent-works/restaurant-rec ommendations-groups-people/, 2016.

[15] Daniel Tuan. Recommender systems-how they works and their impacts.

http://findoutyourfavorite.blogspot.sn/2012/04/content-based-f iltering.html, 2015.

[16] John S. Breese, David Heckerman, and Carl Kadie. Empirical analysis of predictive algorithms for collaborative filtering. in Proceedings of the Fourteenth Conference on Uncertainty and Artificial intelligence, UAI'98, pages 43-52, SanFrancisco, CA, USA, 1998. Morgan Kaufmann Publishers nc. 
[17] R. Deckeriand H. J. (Eds.) Lenz, editors. Advances n Data Analysis: Proceedings of the $30^{\text {th }}$ Annual Conference of The Gesellschaft FA 1 1iriKlassif kation EV, Freie Universitat Berlin, March 8-10. Springer Science and Business Media, 2006.

[18] Paul Resnick, Neophytos acovou, Mitesh Suchak, Peter Bergstrom, and John Riedl. Grouplens: an open architecture for collaborative filtering of net news. in Proceedings of the 1994 ACMiconference on Computer supported cooperative work, pages 175-186. ACM, 1994.

[19] Upendra Shardanand and Pattie Maes. Social nformation filtering: algorithms for automating 'word of mouth'. in Proceedings of the SIGCHI conference on Human factors and computing systems, pages 210-217. ACM Press/Addison-Wesley Publishing Co., 1995.
[20] Shah Khusro, Zafar Ali, and irfan Ullah. Recommender Systems: issues, Challenges, and Research Opportunities, pages 1179-1189. Springer Singapore, Singapore, 2016.

[21] Malinowski J, KeimT, Wietzel T (2005). Analyzing the Impact of IS Support on Recruitment Processes: An E-recruitment Phase Model. In Proceedings of the ninth Pacific Asia conference on information systems (PACIS-2005)), Bangkok, Thailand pp. 977-988.

[22] Belkhirat A, Belkhir A, Bouras A (2011). A New Similarity Measure for the Profiles Management. UKSim 13th International Conference on Modelling and Simulation. Cambridge, United Kingdom: IEEE. 